\title{
The Link between Right and Left Ventricular Systolic Performance at Rest and after Stress: Insights Into the Mechanism
}

\author{
Dawod Sharif ${ }^{\mathrm{a}, \mathrm{b}, \mathrm{d}}$, Amal Sharif-Rasslan ${ }^{\mathrm{b}}$, Camilia Shahla ${ }^{\mathrm{a}}$, Amin Khalil \\ Uri Rosenschein ${ }^{\mathrm{a}, \mathrm{b}}$, Majed Odeh ${ }^{\mathrm{b}, \mathrm{c}}$
}

\begin{abstract}
Background: Right ventricular (RV) systolic performance is more difficult for evaluation compared to the left ventricle (LV). Despite differences in structure, RV myocardial fibers are in continuity with those of LV. The aim is assessment of the effects of LV wall motion abnormalities (WMA) on RV systolic function at rest and after stress.
\end{abstract}

Methods: Fifty nine subjects, 15 with LV-WMA underwent dobuatmine stress echocardiography (DSE) studies using the usual protocol. Measurement of tricuspid annular plane systolic excursion (TAPSE), velocity (TASV), mitral annular plane systolic excursion (MAPSE) and velocity (MASV), were performed before and immediately after DSE studies.

Results: TAPSE was lower, in those with LV-WMA than in those without, both at rest $20.5 \pm 4.8 \mathrm{~mm}$ versus $24.9 \pm 4.7 \mathrm{~mm}, \mathrm{P}=$ 0.015 and after DSE studies, $21.5 \pm 5.6 \mathrm{~mm}$ versus $27.65 \pm 5.7$ $\mathrm{mm}, \mathrm{P}=0.005$. DSE studies did not change TAPSE significantly in the presence of LV-WMA. TASV at rest in those with LV-WMA was $16.5 \pm 2.7 \mathrm{~cm} / \mathrm{sec}$ and similar to that in those without, $17.6 \pm$ $3 \mathrm{~cm} / \mathrm{sec}$. In both groups the velocity increased after DSE studies, $23.25 \pm 7.5 \mathrm{~cm} / \mathrm{sec}, \mathrm{P}=0.01$ with LV-WMA, and $27.5 \pm 6 \mathrm{~cm} / \mathrm{sec}$, $\mathrm{P}=0.0005$, without LV-WMA. Despite similar TASV at rest, the TAPSE/TASV ratio, indicating duration of shortening, was lower $(124 \pm 21 \mathrm{msec})$ in subjects with of LV-WMA, than in those without (145 $\pm 27 \mathrm{msec}$ ), $\mathrm{P}=0.0065$, implying increased after load for RV longitudinal shortening in the presence of LV-WMA.

Conclusions: TAPSE is lower at rest and after DSE studies in sub-

\footnotetext{
Manuscript accepted for publication September 24, 2013

${ }^{\mathrm{a}}$ Department of Cardiology, Bnai Zion Medical Center, Haifa, Israel

${ }^{\mathrm{b}}$ Technion - Israel Institute of Technology, Haifa, Israel

${ }^{\mathrm{c}}$ Department of Internal Medicine A, Bnai Zion Medical Center, Haifa,

Israel

${ }^{\mathrm{d}}$ Corresponding author: Dawod Sharif, Department of Cardiology,

Bnai Zion Medical Center, 47 Golomb St, Haifa, Israel.

Email: dawod.sharif@b-zion.org.il
}

doi: http://dx.doi.org/10.4021/cr291w jects with LV-WMA than in subjects without; however, DSE studies increase TPASE only in the absence of LV-WMA. TASV increases after DSE studies and is similar at rest in both groups with or without LV-WMA. It seems that LV-WMA increases after load to RV longitudinal motion.

Keywords: Echo-Doppler; Systolic function; Contraction reserve; Dobuatmine

\section{Introduction}

Evaluation of left ventricle (LV) systolic function by transthoracic echocardiography is standardized and widely applied. Previously, the dynamics of changes in longitudinal LV systolic function induced by stress have been reported [1]. On the other hand, right ventricular (RV) geometry is complex and as a result, it is often not adequately evaluated. When attempted, frequently, RV systolic function is evaluated by the eye-balling method. Objective evaluation of systolic RV function may be achieved by using the systolic percent area change [2], or more recently, by using threedimensional echocardiography [3].

Longitudinal systolic performance abnormalities precede LV systolic radial abnormalities [4]. Tricuspid annular plane systolic excursion (TAPSE) may be applied to estimate $\mathrm{RV}$ systolic performance $[2,5,6]$. In addition, RV muscle fibers are linked to those of LV [7, 8]. Therefore, the present study was performed in order to evaluate the effects of $\mathrm{LV}$ wall motion abnormality (WMA) on RV systolic function and reserve, and to explore the possible mechanisms of the interaction between $\mathrm{LV}$ and $\mathrm{RV}$.

\section{Methods}

\section{Population}

Fifty eight patients, 29 women, age $60.3 \pm 13.4$ years, were prospectively evaluated for the presence of coronary artery disease. Fifteen of the subjects had LV-WMA at rest. Pa- 
Table 1. RV Longitudinal Systolic Function Parameters at Rest and After DSE Studies in Normal Subjects With and in Those With LV-WMA Only After DSE

\begin{tabular}{lllll}
\hline & & REST & DSE & P \\
& & & \\
& & & \\
TAPSE $(\mathrm{mm})$ & WMA after DSE & $24.04 \pm 4.2$ & $27.2 \pm 5.9$ & 0.13 \\
& NO-WMA & $24.9 \pm 4.8$ & $27.7 \pm 6.2$ & 0.062 \\
& P & 0.556 & 0.818 & \\
TASV $(\mathrm{cm} / \mathrm{sec})$ & WMA after DSE & $16.9 \pm 4.1$ & $26.4 \pm 8.3$ & 0.0007 \\
& NO-WMA & $17.6 \pm 3.4$ & $27.5 \pm 7.2$ & $1.37 \times 10^{-11}$ \\
TAPSE/ & P & 0.59 & 0.64 & \\
TASV $(\mathrm{msec})$ & WMA after DSE & $147.6 \pm 33.6$ & $108 \pm 23.3$ & 0.0073 \\
& NO-WMA & $143.4 \pm 29.3$ & $102.6 \pm 22.5$ & $3.3 \times 10^{-7}$ \\
& P & 0.82 & 0.105 & \\
& WMA after DSE & $70.3 \pm 8.8$ & $135.5 \pm 14.3$ & $5.2 \times 10^{-15}$ \\
& NO-WMA & $70.7 \pm 9.96$ & $139.6 \pm 9.9$ & $3.08 \times 10^{-44}$
\end{tabular}

tients with pulmonary disease, right heart disease, valvular heart disease, atrial fibrillation or other rhythm disturbances and acute coronary syndrome within 3 months were not included. All underwent dobutamine stress echocardiography (DSE) studies using the usual protocol and measurement of $\mathrm{RV}$ and LV systolic function before and after DSE studies.

\section{Dobutamine stress echocardiography}

The protocol of dobutamine infusion consisted of 3 minute stages for each dose, starting with $5 \mu \mathrm{g} / \mathrm{kg} / \mathrm{min}$ and increasing to $10,20,30$ and $40 \mu \mathrm{g} / \mathrm{kg} / \mathrm{min}$. If end-points did not occur or $85 \%$ of the age adjusted heart rate was not achieved, $0.25 \mathrm{mg}$ atropine was injected every 2 minutes up to $1 \mathrm{mg}$ or until the target heart rate was achieved. Blood pressure and 12 lead electrocardiograms were recorded at rest and throughout the DSE study. Horizontal or down-sloping $>1$ mm ST-segment depression at $0.06 \mathrm{sec}$ after the J point were considered as evidence for myocardial ischemia.

\section{Image acquisition}

Images were obtained while the patients in the left lateral decubitus position. A standard commercial Siemens, Acuson Sequoia echocardiography system, California, equipped with 3.5 MHZ transducers was used. All patients had complete Doppler echocardiographic studies before DSE. Parasternal long axis and short axis, as well as, apical 4-chamber and 2-chamber views were recorded at rest, low dose dobutamine infusion, peak exercise and in the recovery period. Digital images were stored on magneto-optic discs for later off-line analysis. In addition super VHS videotape recordings were performed throughout the studies.

\section{Dobutamine stress echocardiographic analysis}

Segmental LV wall motion analysis was performed using 16-segment model [7]. Regional wall motion and segmental score was estimated as normal $=1$, hypokinetic $=2$, akinetic $=3$ or dyskinetic $=4$. New or worsening segmental wall motion was considered as ischemic response. Ischemic response (I) was identified when wall motion decreased by at least 1 grade in 2 adjacent segments or wall motion decreased by at least 2 grades in 1 segment, otherwise no ischemia, or normal response $(\mathrm{N})$ was diagnosed. Wall motion score index (WMSI) was calculated as: WMSI $=($ Sum of scores of 16 segments)/16.

\section{Longitudinal RV and LV systolic function}

Measurements of TAPSE (mm) using M-mode echocardiography at the lateral border of the tricuspid annulus from apical four chamber view, and TASV $(\mathrm{cm} / \mathrm{sec})$ from the same approach and site using pulsed-wave tissue Doppler imaging were performed. Mitral annular plane systolic excursion (MAPSE) from M-mode echocardiography ( $\mathrm{mm}$ ) of the mitral annulus at the lateral and septal borders, and mitral annular systolic velocity (MASV) from the same sites using pulsed-wave tissue Doppler imaging $(\mathrm{cm} / \mathrm{sec})$ were performed. Ratios of TAPSE/MAPSE and TASV/MASV were calculated. Measurements and calculations were performed at rest and immediately after DSE. 
Table 2. RV Longitudinal Systolic Function Parameters at Rest and After DSE Studies in Subjects With and Without LV-WMA at Rest

\begin{tabular}{|c|c|c|c|c|}
\hline & & REST & DSE & $\mathbf{P}$ \\
\hline \multirow[t]{3}{*}{ TAPSE (mm) } & WMA & $20.5 \pm 4.8$ & $21.5 \pm 5.6$ & 0.3 \\
\hline & NO-WMA & $24.5 \pm 4.5$ & $27.5 \pm 5.7$ & 0.006 \\
\hline & $\mathrm{P}$ & 0.0088 & 0.002 & \\
\hline \multirow[t]{3}{*}{ TASV $(\mathrm{cm} / \mathrm{sec})$} & WMA & $16.5 \pm 2.7$ & $23.3 \pm 7.5$ & 0.0057 \\
\hline & NO-WMA & $17.3 \pm 3.4$ & $27.1 \pm 6.8$ & $1.37 \times 10^{-11}$ \\
\hline & $\mathrm{P}$ & 0.2 & 0.06 & \\
\hline \multirow[t]{3}{*}{ TAPSE/TASV (msec) } & WMA & $124 \pm 21$ & $96 \pm 19$ & 0.0019 \\
\hline & NO-WMA & $145 \pm 27$ & $105 \pm 22$ & $5.01 \times 10^{-10}$ \\
\hline & $\mathrm{P}$ & 0.0065 & 0.105 & \\
\hline \multirow[t]{3}{*}{ HR (bpm) } & WMA & $74.2 \pm 17.6$ & $130.9 \pm 15.6$ & $4.35 \times 10^{-10}$ \\
\hline & NO-WMA & $70.7 \pm 9.96$ & $138 \pm 11.9$ & $3.08 \times 10^{-44}$ \\
\hline & $\mathrm{P}$ & 0.48 & 0.123 & \\
\hline
\end{tabular}

\section{Statistical analysis}

Mean values and standard deviation of TAPSE, MAPSE, TASV, MASV and ratios were calculated for all subjects. Student t-test assuming unequal variances, was performed; $\mathrm{P}<0.05$ was considered significant. Comparison was performed between subjects with and those without LV-WMA. Comparisons between RV and LV findings were performed using student t-test.

\section{Results}

All subjects underwent DSE studies safely and uneventfully. In subjects without LV-WMA, heart rate increased from 70.7 $\pm 10 \mathrm{bpm}$ to $138 \pm 11.8 \mathrm{bpm}, \mathrm{P}<0.00001$, and in subjects with LV-WMA increased from $74.2 \pm 17.6 \mathrm{bpm}$ to 130.9 $\pm 15.6 \mathrm{bpm}, \mathrm{P}<0.00001$. Heart rate was similar between groups both at rest and after DSE studies. Systolic blood pressure increased from $138 \pm 7 \mathrm{mmHg}$ to $162 \pm 9 \mathrm{mmHg}, \mathrm{P}$ $<0.0001$, in all subjects without significant inter-group difference.

\section{Left ventricular wall motion}

In 26 subjects WMSI was 1 at rest and did not change after DSE studies, while in 18 subjects WMSI was 1 at rest and increased to $1.12 \pm 0.14$ after stress. In 15 subjects WMSI at rest was $1.36 \pm 0.34$ and increased to $1.47 \pm 0.32$ after DSE studies.

\section{Tricuspid annular systolic excursion (TAPSE) and veloc- ity (TASV)}

TAPSE and TASV were similar at rest and after DSE in normal subjects and in those with LV-WMA only after DSE (Table 1). Therefore, further analyses and comparisons were performed between subjects with LV-WMA at rest and those without resting LV-WMA.

In subjects with LV-WMA at rest, TAPSE was lower than in those without resting LV-WMA, both at rest and after DSE, indicating lower RV systolic function (Table 2). While in subjects with resting LV-WMA, no significant change in TAPSE was found after DSE; in those without resting LVWMA, TAPSE increased significantly after DSE, implying good RV systolic function reserve. Contrary to TAPSE, TASV was similar in both groups at rest and after DSE studies (Table 2). TASV increased during DSE in both groups with or without resting LV-WMA, but more significantly in those without LV-WMA at rest. Despite similar heart rate in both groups (Table 2), effective average tricuspid systolic displacement time as measured by TAPSE/TASV was larger at rest, but not after DSE studies, in those without LV-WMA at rest (Table 2). After DSE studies the increase in TASV 
Table 3. Relationship Between Longitudinal RV and LV Ventricular Systolic Function Parameters at Rest and After DSE Studies in Subjects With and Without LV-WMA at Rest

\begin{tabular}{|c|c|c|c|c|c|}
\hline & & & REST & DSE & $\mathbf{P}$ \\
\hline \multirow[t]{6}{*}{ TAPSE/MAPSE } & IVS & WMA & $2.27 \pm 1.02$ & $2.08 \pm 0.87$ & 0.65 \\
\hline & & NO-WMA & $1.87 \pm 0.36$ & $2.0 \pm 0.5$ & 0.25 \\
\hline & & $\mathrm{P}$ & 0.247 & 0.77 & \\
\hline & LATERAL & WMA & $1.62 \pm 0.46$ & $1.64 \pm 0.55$ & 0.93 \\
\hline & & NO-WMA & $1.62 \pm 0.39$ & $1.72 \pm 0.5$ & 0.28 \\
\hline & & $\mathrm{P}$ & 0.95 & 0.66 & \\
\hline \multirow[t]{6}{*}{ TASV/MASV } & IVS & WMA & $1.68 \pm 0.74$ & $1.81 \pm 0.46$ & 0.62 \\
\hline & & NO-WMA & $1.61 \pm 0.34$ & $1.5 \pm 0.3$ & 0.155 \\
\hline & & $\mathrm{P}$ & 0.76 & 0.058 & \\
\hline & LATERAL & WMA & $1.77 \pm 0.93$ & $1.82 \pm 0.58$ & 0.89 \\
\hline & & NO-WMA & $1.52 \pm 0.43$ & $1.58 \pm 0.47$ & 0.53 \\
\hline & & $\mathrm{P}$ & 0.38 & 0.21 & \\
\hline
\end{tabular}

was associated with shortening of TAPSE/TASV, more so in those without resting LV-WMA (Table 2).

\section{Relation of RV and LV systolic function and reserve}

RV longitudinal systolic function as measured by TAPSE was coordinated with LV septal and lateral excursions both at rest and after DSE studies. Thus, TAPSE/MAPSE did not change after DSE studies (Table 3). Moreover, the coordination between RV and LV systolic displacements was maintained in subjects with or without LV-WMA (Table 3).

In addition, TASV was coordinated with that of LV. Presence or absence of LV-WMA did not affect this interventricular coordination both at rest and after DSE studies (Table 3).

As depicted in Table 3, RV longitudinal systolic displacement and velocities were higher than those of LV. However, lateral TAPSE, despite being larger than lateral MAPSE, their association was closer than that with septal MAPSE (Table 4). These septal-lateral differences were evident at rest in those with or without LV-WMA; however, after DSE this difference disappeared in the presence of LV-WMA (Table 4). Rate of change of longitudinal systolic displacements-TASV and MASV did not show these septal-lateral LV differences in relation to $\mathrm{RV}$, both at rest and after DSE studies, irrespective of presence or absence of LV-WMA (Table 4).

\section{Discussion}

In this study it was shown that RV systolic function at rest and after DSE as measured by TAPSE was lower in those with LV-WMA at rest compared to those without. In addition, RV systolic function reserve as evaluated by the increase in TAPSE after DSE in normal subjects, was abolished in the presence of resting LV-WMA.

As we found, measurement of TAPSE is feasible and easy to obtain, and the values are similar to those found in a previous study which proved the accuracy of this parameter in assessing RV systolic function compared to other parameters like fractional area change [2]. Another study also found excellent correlation between TAPSE and RV fractional area change [9]. TAPSE predicted survival in patients with pulmonary hypertension [10]. However, TAPSE showed a poor correlation with RV ejection fraction in the presence of severe tricuspid regurgitation [11]. Moreover, RV systolic function is not the sole determinant of TAPSE [12]. Similar to our findings, others reported that TAPSE is affected by LV systolic function, and added significant information to prognosis in patients with heart failure $[13,14]$. Thus our TAPSE-findings confirmed those reported previously as detailed above, but in addition we report also the limited increase in TAPSE in the presence of resting LV-WMA.

To answer the question why RV systolic function is affected by LV systolic function abnormalities, understanding 
Table 4. Comparison of Ratios of RV to LV Ventricular Septal and Lateral Systolic Function Parameters in Subjects With and Without LV-WMA at Rest

\begin{tabular}{llll}
\hline & & \multicolumn{2}{c}{ P(IVS:LATERAL) } \\
\cline { 3 - 3 } & & Rest & DSE \\
\hline \multirow{2}{*}{ TAPSE/MAPSE } & WMA & $0.0753(0.038)$ & $0.172(0.086)$ \\
TASV/MASV & NO-WMA & $0.0016(0.0008)$ & $0.0153(0.0076)$ \\
& WMA & $0.78(0.39)$ & $0.96(0.48)$ \\
& NO-WMA & $0.33(0.16)$ & $0.36(0.18)$ \\
\hline
\end{tabular}

$P$ value statistics two tailed and one tailed (in brackets) student test.

the anatomy and relation of the myocardial fibers of both ventricles is essential. Thus, contrary to the left ventricle which is composed of three myocardial fiber layers-superficial obliquely directed fibers, middle circumferentially directed fibers and deep inner layer with longitudinal fibers; the RV wall is composed only of two myofiber layers [15]. The direction of RV myocardial fibers of the superficial layer is circumferential parallel to the atrio-ventricular groove and turns oblique toward the apex, and is continuous with the superficial layer of the LV [16], while the inner myocardial layer of the RV is longitudinally directed from base to apex. Therefore, it seems that the arrangement of myocardial fibers and the continuity between RV and LV myocardial fibers are responsible for the decrease in RV systolic function and reserve, as assessed by TAPSE, in the presence of LV-WMA.

A previous study found that TASV correlated with fractional area change of RV and with TAPSE [9]. Yet, another study found that TASV did not correlate with RV ejection fraction in the presence of severe tricuspid regurgitation [11]. Previously, it was reported that TASV correlated with LV ejection fraction and that $12 \mathrm{~cm} / \mathrm{sec}$ threshold for TASV identified patients with reduced LV systolic function with high specificity [2]. However, in our study, contrary to TAPSE, TASV was similar in both groups and thus was not affected by the presence of LV-WMA. In addition, we found that despite the increase in TASV after DSE, LV-WMA was associated with less increase in TASV compared to those without WMA. It should be emphasized that TASV was similar at rest and after DSE in the presence or absence of LV-WMA.

Why LV-WMA did not affect TASV significantly and yet it decreased TAPSE? In fact, TAPSE is the integral of velocity (TASV) upon time. Despite similar heart rates in the presence or absence of LV-WMA, the ratio of TAPSE/TASV was higher in the absence of LV-WMA. This time index implying longer contraction time of RV myocardial fibers in the absence of LV-WMA can be explained by higher afterload to $\mathrm{RV}$ contraction in the presence of LV-WMA (similar to friction effects with higher deceleration and thus more rapid decrease of velocity). The increase in RV afterload interrupts shortening of RV myocardial fibers prematurely. It seems that regional LV-WMA, by changing geometry may lead to alterations in RV myocardial fibers, and thus may affect contraction period of RV. Increase in pulmonary vascular resistance is another factor which may contribute to these results, however pulmonary hypertension was not found in these patients, and it seems unlikely in this study population. Since TASV was similar in both groups, it can be deduced that LV-WMA did not affect contraction of RV myocardial fibers. Thus, RV contractility seems similar while the afterload impeding contraction of RV myocardial fibers may explain the findings in our study. Since RV size and shape were normal and similar in all subjects, and no significant tricuspid regurgitation was observed, it seems that preload did not affect the results of our study.

The increase in heart rate during DSE studies, significantly shortened effective average TAPSE displacement time. After DSE studies no significant difference in TAPSE/ TASV time index was observed between subjects with and without LV-WMA. It seems that the increase in TASV after DSE contributed to the larger TAPSE post DSE in subjects without LV-WMA.

Changes of TAPSE and TASV paralleled changes in MAPSE and MASV, implying the dependence of RV longitudinal systolic function and reserve on the status of LV. There was no significant difference in the ratio of TAPSE/ MAPSE between the groups at rest and after DSE. In addition, DSE studies did not change this ratio compared to rest in both groups. The ratio TASV/MASV behaved in a similar fashion to TAPSE/MAPSE ratio, and thus emphasizing the interdependence of RV and LV.

In addition to the interventricular septum and pericardium, the continuity between RV and LV myocardial fibers contributes to ventricular interdependence [9]. Despite the position of the interventricular septum, in this study the values of TAPSE were closer to those of lateral LV-MAPSE 
compared to septal-MAPSE especially after DSE, as seen in Table 3. However, TASV/MASV was similar at both septal and lateral regions. This means that the period of septal systolic longitudinal motion is shortened, implying higher afterload to septal longitudinal motion compared to the lateral LV wall.

\section{Limitations}

TAPSE and TASV were similar at rest and after DSE in normal subjects and in those with LV-WMA only after DSE. Therefore, in further analysis, both groups were evaluated as one group and were compared to patients with LV-WMA at rest. It seems that, the WMSI which equaled 1 at rest and increased only to $1.12 \pm 0.14$ after DSE was of small magnitude and therefore the studied RV parameters were normal. It remains to be shown that if WMSI after DSE was higher, the RV parameters would behave abnormally.

\section{Conclusions}

Longitudinal RV systolic performance is related to LV performance, and thus TAPSE is decreased in the presence of LV-WMA. TAPSE increases after DSE studies, however, TPSAE reserve is negligible in the presence of LV-WMA. TASV increases after DSE studies, but was similar in the presence or absence of LV-WMA wall motion abnormality. LV-WMA increases afterload to RV longitudinal systolic performance. Afterload to septal longitudinal motion is higher than to lateral LV and RV systolic motion.

\section{Disclosure}

No contracts, funds or financial supports, and no conflicts of interests.

\section{References}

1. Sahrif D, Rasslan-Sharif A, Shahla C, Rosenschein U. Application of mitral annular systolic displacemt and velocities for the evaluation of left ventricular systolic function and reserve. Cardiol Res. 2011;2(1):36-41.

2. Tamborini G, Pepi M, Galli CA, Maltagliati A, Celeste F, Muratori M, Rezvanieh S, et al. Feasibility and accuracy of a routine echocardiographic assessment of right ventricular function. Int J Cardiol. 2007;115(1):86-89.

3. Kjaergaard J, Petersen CL, Kjaer A, Schaadt BK, Oh JK, Hassager C. Evaluation of right ventricular volume and function by $2 \mathrm{D}$ and $3 \mathrm{D}$ echocardiography compared to MRI. Eur J Echocardiogr. 2006;7(6):430-438.

4. Ballo P, Quatrini I, Giacomin E, Motto A, Mondillo S. Circumferential versus longitudinal systolic function in patients with hypertension: a nonlinear relation. J Am Soc Echocardiogr. 2007;20(3):298-306.

5. Kaul S, Tei C, Hopkins JM, Shah PM. Assessment of right ventricular function using two-dimensional echocardiography. Am Heart J. 1984;107(3):526-531.

6. Germing A, Gotzmann M, Rausse R, Brodherr T, Holt S, Lindstaedt M, Dietrich J, et al. Normal values for longitudinal function of the right ventricle in healthy women $>70$ years of age. Eur J Echocardiogr. 2010;11(8):725728.

7. Fernandez-Teran MA, Hurle JM. Myocardial fiber architecture of the human heart ventricles. Anat Rec. 1982;204(2):137-147.

8. Sanchez-Quintana D, Garcia-Martinez V, Climent V, Hurle JM. Morphological changes in the normal pattern of ventricular myoarchitecture in the developing human heart. Anat Rec. 1995;243(4):483-495.

9. Saxena N, Rajagopalan N, Edelman K, Lopez-Candales A. Tricuspid annular systolic velocity: a useful measurement in determining right ventricular systolic function regardless of pulmonary artery pressures. Echocardiography. 2006;23(9):750-755.

10. Forfia PR, Fisher MR, Mathai SC, Housten-Harris T, Hemnes AR, Borlaug BA, Chamera E, et al. Tricuspid annular displacement predicts survival in pulmonary hypertension. Am J Respir Crit Care Med. 2006;174(9):1034-1041.

11. Hsiao SH, Lin SK, Wang WC, Yang SH, Gin PL, Liu CP. Severe tricuspid regurgitation shows significant impact in the relationship among peak systolic tricuspid annular velocity, tricuspid annular plane systolic excursion, and right ventricular ejection fraction. J Am Soc Echocardiogr. 2006;19(7):902-910.

12. Lopez-Candales A, Rajagopalan N, Saxena N, Gulyasy B, Edelman K, Bazaz R. Right ventricular systolic function is not the sole determinant of tricuspid annular motion. Am J Cardiol. 2006;98(7):973-977.

13. Ghio S, Recusani F, Klersy C, Sebastiani R, Laudisa ML, Campana C, Gavazzi A, et al. Prognostic usefulness of the tricuspid annular plane systolic excursion in patients with congestive heart failure secondary to idiopathic or ischemic dilated cardiomyopathy. Am J Cardiol. 2000;85(7):837-842.

14. Kjaergaard J, Akkan D, Iversen KK, Kober L, Torp-Pedersen C, Hassager C. Right ventricular dysfunction as an independent predictor of short- and long-term mortality in patients with heart failure. Eur J Heart Fail. 2007;9(67):610-616.

15. Ho SY, Nihoyannopoulos P. Anatomy, echocardiography, and normal right ventricular dimensions. Heart. 2006;92(Suppl 1):i2-13.

16. Dell'Italia LJ. The right ventricle: anatomy, physiology, and clinical importance. Curr Probl Cardiol. 1991;16(10):653-720. 